$\mathbb{T}$ periodica polytechnica

\author{
Transportation Engineering \\ $40 / 2(2012) 45+51$ \\ doi: 10.3311/pp.tr.2012-2.01 \\ web: http://www.pp.bme.hu/tr \\ (c) Periodica Polytechnica 2012
}

RESEARCH ARTICLE

\section{Velocity selection by a human driver compared to look-ahead control}

\author{
András Mihály / Németh Balázs / Péter Gáspár
}

Received 2012-10-27

\begin{abstract}
The paper focuses on the design of look-ahead cruise control systems which can adopt the behavior of the driver in the velocity selection process. The automatic system uses information about the oncoming road sections to calculate an economically optimal velocity for the vehicle. This velocity profile may differ greatly from the one selected by a human driver, who only has visual and acoustic information of the oncoming road section. The motivation of the paper is to analyze the behavior of the driver in terms of velocity selection in order to set up a longitudinal driver model. By adopting the driver model in the automatic system's velocity selection process, the motion of the vehicle can be more comfortable for the driver and the passengers of the vehicle, and the traveling time may be closer to that of the human driver.
\end{abstract}

\section{Keywords}

Driver model $\cdot$ look-ahead control $\cdot$ velocity design $\cdot$ energy optimization

\section{András Mihály}

Department of Control for Transportation and Vehicle Systems, BME, Stoczek J. u. 2., H-1111 Budapest, Hungary

e-mail: mihaly.andras@mail.bme.hu

\section{Németh Balázs}

Systems and Control Laboratory, Computer and Automation Research Institute, MTA, Kende u. 13-17., H-1111 Budapest, Hungary

e-mail: bnemeth@sztaki.hu

\section{Péter Gáspár}

Systems and Control Laboratory, Computer and Automation Research Institute, MTA, Kende u. 13-17., H-1111 Budapest, Hungary

e-mail: gaspar@sztaki.hu

\section{Introduction}

Today's vehicles equipped with conventional cruise control systems are able to maintain steady speed set by the driver by adjusting the longitudinal control forces acting on the vehicle, i.e activating the throttle or the brake. Nowadays adaptive cruise control systems are becoming widespread among premium and middle class cars. This device enables the vehicle to follow the speed set by the driver and if the lane is occupied, it follows preceding vehicles automatically at a predefined safe distance. However, these controllers do not have information about velocity regulations and inclinations of the oncoming road sections, thus the selected velocity of the vehicle is based on instantaneous effects. In this manner, the velocity selected by the automatic system is not optimal in terms of economy and emission. Nevertheless, in the state-of-the-art automotive applications, comfort and economy are major objectives, see [1].

In the paper for the design of the vehicle's velocity a lookahead control method is proposed, in which the road inclinations and speed limits are taken into consideration. In this method the assumption is that information about the actual and oncoming road is available, such as speed limits and road inclinations. By choosing an optimal velocity the number of unnecessary accelerations and brakings can be reduced, thus energy required by the actuators and fuel consumption can be reduced significantly. Several methods in which road inclinations are taken into consideration have already been proposed, see [2,3]. In [4] the approach was evaluated in real experiments where the road slope was estimated in [5].

On a given route, the velocity proposed by the look-ahead control system may differ greatly from the velocity selected by the human driver. This is due to the fact that besides the different behaviors in terms of following the speed limit, the driver has limited and inaccurate information about the forthcoming road section. The driver's vision and the estimation of the road inclination ahead are both limited, thus an optimal velocity is hard if not impossible to reach. On the other hand, the automatic system can select velocity in coherence with the oncoming road, for example moderate the velocity in advance of a slope or a speed limit sign. 
The paper focuses on investigating the behaviors of different drivers compared to an automatic adaptive control system presented in [6,7]. In the simulation section a motorway route with real data is analyzed for both cases. The evaluation of the simulation is performed with Matlab Simulink using Carsim simulation environment. For the realistic mapping of the driver's behavior, a hardware-in-the-loop simulation system is used. With measured data of both the automatic system and the driver the velocity selection can be compared as well as the total energy consumption. It will be shown in the paper that with the lookahead control significant energy can be saved with relatively little increase in the traveling time. However, as for all automatic driving aids, the cruise control system must provide a comfortable feel for the vehicle's driver and passengers. For enhancing passenger comfort the automatic system's weighting can be tuned to be closer to that of the human driver's. In this way, a good balance can be achieved between economy and passenger comfort.

This paper is organized as follows. In Section 2 the driver simulation environment is presented. In Section 3 the relation between velocity and road slopes is analyzed. In Section 4 the relation between the driver model and road slopes is analyzed. In Section 5 the simulation results are summarized. Finally, in Section 6 the concluding remarks are summarized.

\section{Driver simulation environment}

Fig. 1 shows the simulator with a real car connected to a simulation environment. For simulation purposes, the control of the vehicle's communication network has been taken over by the simulator unit.

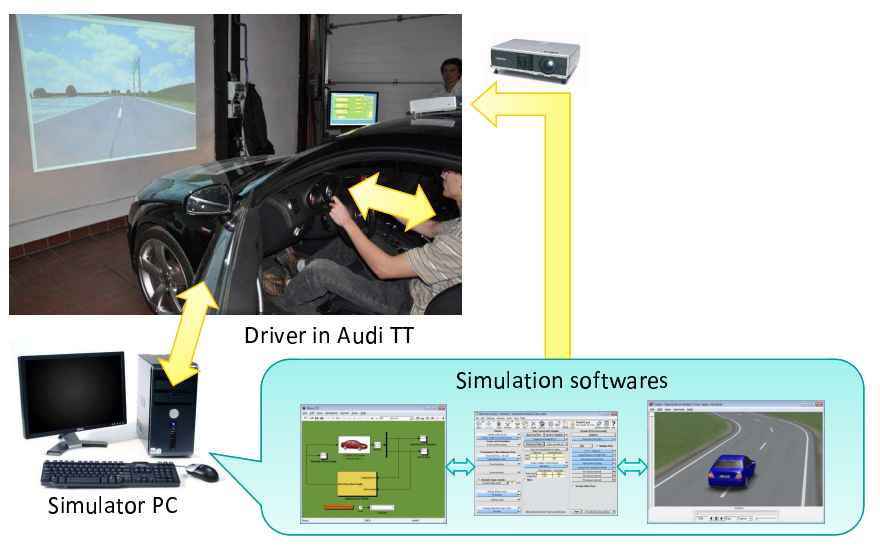

Fig. 1. Architecture of driving simulator

The simulation environment contains HMI (Human Machine Interface), a high-accuracy validated simulation software operated on a PC and a visual system with real-time graphics. The specific signals for the simulation (the position of the accelerator and the brake pedal along with the steering angle) are read through the CAN network by using standard communication interface. The driver can induce various vehicle maneuvers by using the steering wheel and the accelerator/brake pedals of the car. Based on the excitations the validated simulation software generates the signals of the vehicle during simulation.

The Driving Simulator of CarSim shows the vehicle maneuvers by real-time graphics projected in front of the vehicle and it provides the signals during the journey. The standing vehicle can be driven almost exactly the same way as in real life: there is engine sound and screech while skidding; the dash panel displays the current speed and revolution and one can shift gears just like in real life.

Various journey scenarios can be generated by the simulation system. The advantage of the system is that besides measuring various signals, i.e., the steering angle, the positions of the accelerator and the brake pedal or the gear level, in principle any signals can be monitored during the simulations. In this way signals, which are not measurable in practice, can be achieved for identification purposes.In the test scenarios various routes with real data can be loaded in the Driving Simulator. The data contains both the terrain characteristics and geographical information such as height data, speed limits. In the simulation procedure the driver is able to drive along the road section while the vehicle signals are measured, saved and post-processed. Based on the responses of different drivers to the effects of disturbances, speed limits, road slopes can be analyzed.

\section{Relation between velocity and road slopes}

The relationship between the optimal velocity and the road inclinations was introduced in [10]. The route of the vehicle can be divided into $n$ sections using $n+1$ number of points. The division of the route is not necessarily of equal lengths. The rates of the inclinations of the road and those of the speed limits are assumed to be known at the endpoints of each section. The velocity at section point $j$ should reach a predefined reference velocity $v_{\text {ref }, j}^{2} j \in[1, n]$, which is usually the maximum velocity of the vehicle (speed limit). It is also an important goal to track the momentary value of the velocity, which is formalized in the following form: $\dot{\xi}_{0}^{2} \rightarrow v_{\text {ref, } 0}^{2}$. The velocity of the $n^{\text {th }}$ section point is the following:

$$
\dot{\xi}_{n}^{2}=\dot{\xi}_{0}^{2}+\frac{2}{m} s_{1} F_{l 1}-\frac{2}{m} \sum_{i=1}^{n} s_{i} F_{\mathrm{di}}
$$

The $F_{\text {di }}$ disturbance force can be divided in two parts: the first part is the force resistance from road slope $F_{d i, r}=m g \sin \alpha_{i}$, while the second part $F_{d i, o}$ contains all of the other resistances such as rolling resistance, aerodynamic forces etc.

Velocities of the vehicle at section points are calculated from (1) in the following way:

$$
\dot{\xi}_{0}^{2}+\frac{2}{m} s_{1}\left(F_{l 1}-F_{d 1, o}\right)=v_{\mathrm{ref}, n}^{2}+\frac{2}{m} \sum_{i=1}^{n} s_{i} F_{\mathrm{di}, \mathrm{r}}
$$

In the next step a weight $Q$ is applied to the momentary (initial) velocity and weights $\gamma_{1}, \gamma_{2}, \ldots, \gamma_{n}$ are applied to the reference velocities of the road sections in advance. The weights 


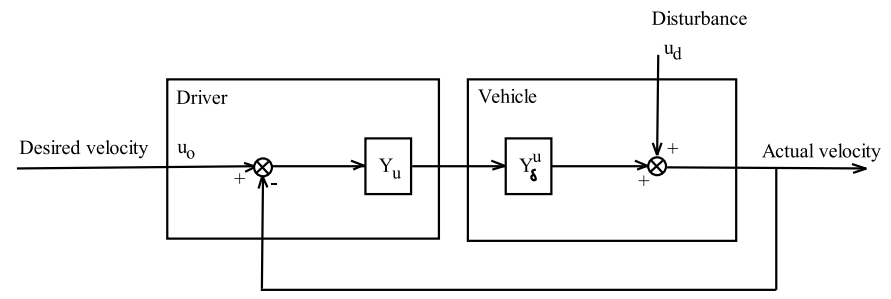

Fig. 2. Model for velocity control

should sum up to one, i.e., $\gamma_{1}+\gamma_{2}+\ldots+\gamma_{n}+Q=1$. While the weights $\gamma_{i}$ represent the rate of the road conditions, weight $Q$ determines the tracking requirement of the momentary reference velocity $v_{\text {ref }, 0}$.

$$
\gamma_{i} \dot{\xi}_{0}^{2}+\frac{2}{m} s_{1} \gamma_{i}\left(F_{l 1}-F_{d 1, o}\right)=\gamma_{i} v_{\mathrm{ref}, 1}^{2}+\frac{2}{m} s_{1} \gamma_{1} F_{d 1, r}
$$

Note that weights have an important role in control design. By making an appropriate selection of the weights $Q$ and $\gamma_{i}$ the importance of the road condition is considered. Taking the weights into consideration the following formula is yielded:

$$
\dot{\xi}_{0}^{2}+\frac{2 s_{1}}{m}(1-Q)\left(F_{l 1}-F_{d 1, o}\right)=\vartheta
$$

where value $\vartheta$ depends on the predicted road slopes, the reference velocities and the prediction weights

$$
\vartheta=Q v_{\mathrm{ref}, 0}^{2}+\sum_{i=1}^{n} \gamma_{i} v_{\mathrm{ref}, i}^{2}+\frac{2}{m} \sum_{i=1}^{n}\left(s_{i} F_{\mathrm{di}, \mathrm{r}} \sum_{j=i}^{n} \gamma_{j}\right)
$$

In the final step a control-oriented vehicle model, in which reference velocities and weights are taken into consideration, is constructed. The momentary acceleration of the vehicle is expressed in the following way: $\ddot{\xi}_{0}=\left(F_{l}-F_{d, o}-F_{d 1, r}\right) / m$ where $F_{d 1, r}=m g \sin \alpha$. Eq. (4) is rearranged:

$$
\dot{\xi}_{0}=\lambda
$$

where the parameter $\lambda$ is calculated in the following way based on the designed $\vartheta$ :

$$
\lambda=\sqrt{\vartheta-2 s_{1}(1-Q)\left(\ddot{\xi}_{0}+g \sin \alpha\right)} .
$$

The aim of the control design is to minimize the longitudinal force in order to reduce the energy required by traveling. The longitudinal force $\left(F_{l 1}\right)$ can be expressed as the linear function of weights $Q$ and $\gamma_{i}$ based on equation (6):

$$
F_{l 1}=\beta_{0}(Q)+\beta_{1}(Q) \gamma_{1}+\beta_{2}(Q) \gamma_{2}+\ldots+\beta_{n}(Q) \gamma_{n}
$$

where $\beta_{i}$ are the coefficients of $\gamma_{i}$, and they depend on prediction weight $Q$. In practice, a quadratic form is used. This minimization problem is met by the transformation of the quadratic form with the following constrains:

$$
\begin{aligned}
& \bar{F}_{l 1}^{2}\left(Q, \gamma_{i}\right)=\left(\beta_{0}(Q)+\beta_{1}(Q) \gamma_{1}+\ldots+\beta_{n}(Q) \gamma_{n}\right)^{2} \\
& 0 \leq Q, \gamma_{i} \leq 1 \text { and } Q+\sum \gamma_{i}=1
\end{aligned}
$$

This task is a nonlinear optimization problem because of the prediction weights.

\section{Relationship between the driver model and road slopes}

\subsection{Optimization method}

Unlike the automatic cruise control system, the human driver only has visual information about the road. The driver's visual perception of the road ahead is much shorter than the road known by the automatic system, and the human driver can only approximate the road inclinations. In a conventional vehicle without cruise control the driver selects the vehicle's velocity based on the road and traffic conditions. In another vehicle, in which the proposed look-ahead method is applied, the selected velocities are calculated based on the optimization procedure. In the method the weighting factors are also the results of the optimization procedure.

However, based on the relationship between the weighting factors and the selected velocities, the weighting factors set by the human driver intensively can be calculated from a conventionally-driven vehicle as well. Measuring the driver's velocity, acceleration and position data on a given road (with known terrain characteristics), it is possible to regressively calculate the weighting factors. In this manner the weighting factors are compared to the weights calculated by the automatic system, thus the latter can be modified to adapt to the driver's behavior. Moreover, the weights of various drivers can also be compared to each other and to the automatic system as well.

The regressive calculation of the driver's weight is derived as follows. Equations in (2) contain the velocities of the vehicle at section points $i=[0,1 \ldots n]$. These equations are multiplied by weighting factors $Q, \gamma_{i}$. The right-hand side of these equations can be written as:

$$
\vartheta=Q v_{\text {ref }, 0}^{2}+\Omega \Gamma
$$

where

$$
\begin{aligned}
& \Omega=\left[\begin{array}{c}
v_{\mathrm{ref}, 1}^{2}+\frac{2}{m} s_{1} F_{d 1, r} \\
v_{\mathrm{ref}, 2}^{2}+\frac{2}{m} \sum_{i=1}^{2} s_{i} F_{\mathrm{di}, \mathrm{r}} \\
\vdots \\
v_{\mathrm{ref}, n}^{2}+\frac{2}{m} \sum_{i=1}^{n} s_{i} F_{\mathrm{di}, \mathrm{r}}
\end{array}\right]^{T}, \\
& \Gamma=\left[\begin{array}{lll}
\gamma_{1} & \ldots & \gamma_{n}
\end{array}\right]^{T}
\end{aligned}
$$

The left hand side of (2) can be transformed using the following relation between acceleration and the forces acting on the vehicle:

$$
\ddot{\xi}_{0}=\left(F_{l}-F_{d, o}-F_{d 1, r}\right) / m
$$

where $F_{d 1, r}=m g \sin \alpha$. After organizing equation (6) and substituting the above formula, the following equation is derived to determine the velocity of the vehicle:

$$
\vartheta=\dot{\xi}_{0}^{2}+2 s_{1}(1-Q)\left(\ddot{\xi}_{0}+g \sin \alpha\right)
$$

Next equations (10) and (13) are combined:

$$
\dot{\xi}_{0}^{2}+2 s_{1}(1-Q)\left(\ddot{\xi}_{0}+g \sin \alpha\right)=Q v_{\mathrm{ref}, 0}^{2}+\Omega \Gamma
$$


Note that in the above equation $\dot{\xi}_{0}, \ddot{\xi}_{0}$ and $\alpha$ are measured signals of the driver's simulation, while $\vartheta$ is calculated with the unknown weighting parameters. The optimization task is to minimize function $f$ defined with equation (14) as follows:

$$
f=\dot{\xi}_{0}-\sqrt{Q v_{\text {ref }, 0}^{2}+\Omega \Gamma-2 s_{1}(1-Q)\left(\ddot{\xi}_{0}+g \sin \alpha\right)}
$$

with the constraints $Q+\sum \gamma_{i}=1$ and $0<Q, \gamma_{i}<1$.

The determination of the possible weights of the driver is evaluated as follows: First, weight $Q$ is set to a constant. Second, the matrix $\Gamma$ is computed with the above defined optimization procedure, using the measured signals from the driver's simulation. Third, the computed $Q, \gamma_{i}$ is applied to simulate vehicle dynamics using the driver model. Fourth, the measured and simulated signals are compared. All of these steps are accomplished for different $Q$ values. Finally, the set $Q, \gamma_{i}$, which minimizes the differences between the measurement and the simulation, is chosen. Note that value of $Q$ can be dynamically changing during the travel of the vehicle, but for numerical reasons we assume $Q$ to be constant.

\subsection{Driver model}

Another method for the reconstruction of the driver's weight selection is the following. It is assumed that in the velocity selection process the driver tries to follow the regulated maximum velocity, and only considers instantaneous effects such as disturbances acting on the vehicle, where $\gamma_{i}$ values are chosen to be zero. For the further analysis, a driver model introduced in [9] is used to capture the behavior of the driver in terms of following the desired velocity. This linearized model assumes that the driver perceives and operates only on forward velocity, and the dynamic model of the vehicle is known. The scheme of this driver model is shown in Fig. 2. In this model, the driver uses the accelerator pedal for speed regulation, and tries to maintain a constant speed in the presence of speed disturbances resulting from road slopes, aerodynamic and road resistances.

In this driver model $Y_{u}$ represents the transfer function of the driver, while $Y_{\delta}^{u}$ is a transfer function of the vehicle relating forward speed to accelerator pedal position. This can be approximated by:

$$
Y_{\delta}^{u}=\frac{K_{\delta}^{u}}{\left(T_{u} s+1\right)}
$$

where $T_{u}$ is a time constant associated with the change of vehicle speed, while $K_{\delta}^{u}$ is associated with the accelerator pedal sensitivity. For the simulation in Carsim, the above transfer function is used with $T_{u}=10$ and $K_{\delta}^{u}=1$. The following model is used to capture the driver's behavior:

$$
Y_{u}=K_{u}\left(\frac{1}{s}+T_{L}\right) e^{-s \tau}
$$

A representative set of driver parameters is used for the simulation: $K_{u}=0.3 ; T_{L}=12 ; \tau=1.7$.

The task is to define the constantly changing $Q_{i}$ weights used by the driver, which can be calculated on-board during the journey of the vehicle. Assuming that the vehicle dynamics and the

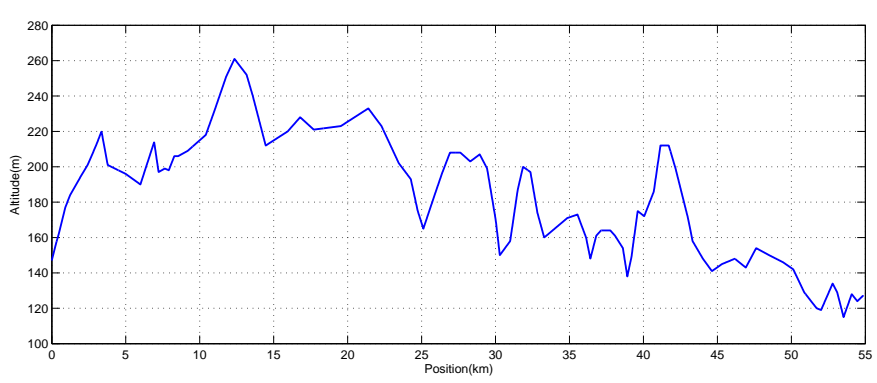

(a) Terrain characteristics

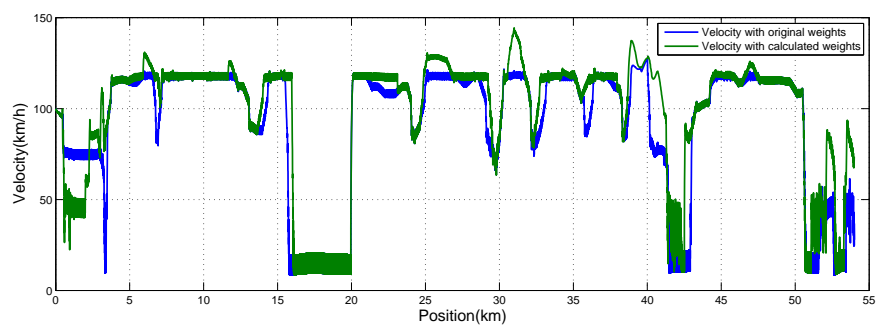

(b) Comparison of the original and the simulated velocity

Fig. 3. Validation of the optimization method

driver's function are known along with the actual reference velocity and the road slope, it is possible to calculate the velocity which the driver would have chosen in the presence of the actual disturbances. After substituting $\Gamma=0$ in (14) and rearranging the equation, weight $Q_{i}$ can be expressed as follows:

$$
Q_{i}=\frac{\dot{\xi}_{0}^{2}+2 s_{1} \ddot{\xi}_{0}+2 s_{1} g \sin \alpha}{v_{\mathrm{ref}, 0}^{2}+2 s_{1} \ddot{\xi}_{0}+2 s_{1} g \sin \alpha}
$$

where $\dot{\xi}, \ddot{\xi}$ are calculated with the above driver model, $\alpha$ and $v_{\text {ref }, 0}$ are road information assumed to be known. Thus the automatic look-ahead system can be modified by changing the fixed $Q$ value to that of the calculated $Q_{i}$ value using the driver model. In this way, the optimization process of the look-ahead system can adopt the possible $Q_{i}$ values that the driver may have used in the same section of the route in order to determine $\xi_{i}$ weights. By this method, the velocity profile and the traveling time will be closer to that of the human driver, but energy can still be saved as a result of the optimization considering the information of the oncoming road. The advantage of this method compared to the previously detailed optimization procedure is that the driver's weight $Q_{i}$ adaptation can be realized on-board, thus there is no need to use earlier experiment data for the modification of the automatic system.

\section{Simulation results}

In this section the previously detailed methods are examined with real data motorway simulation in Carsim environment. The terrain characteristics and geographical information are those of the M1 Hungarian highway between Tatabánya and Budapest in a $56 \mathrm{~km}$ long section with several slopes and uphills (see Fig. 3(a)).The regulated maximal velocity is $130 \mathrm{~km} / \mathrm{h}$, but the 
road section contains other speed limits as well (e.g. $80 \mathrm{~km} / \mathrm{h}$ or $100 \mathrm{~km} / \mathrm{h})$.

\subsection{Optimization results}

For the validation of the above described optimization procedure the following experiment was carried out. The vehicle using an automatic look-ahead system was simulated on the Budapest-Tatabánya path, with the $Q$ weight set to zero. In order to carry out the calculation it is necessary to measure the velocity $\left(\dot{\xi}_{0}\right)$ and the longitudinal accelerations of the vehicle $\left(\ddot{\xi}_{0}\right)$ as well as the momentary road inclinations $\alpha$. Next the regressive calculation of the look-ahead system's $\Gamma$ weights was evaluated with the optimization procedure detailed in the previous chapter of this paper. Then the simulation was rerun by substituting the calculated gamma values for those calculated by the look-ahead procedure. The original and the simulated velocity profiles were then compared to each other in Fig. 3 .

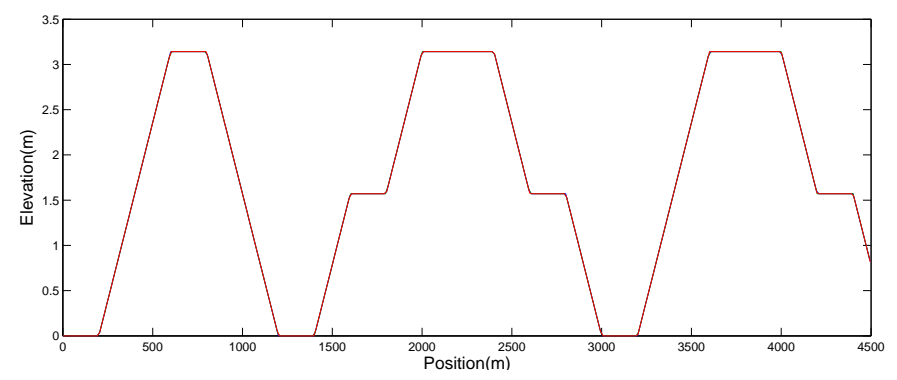

Fig. 4. Simulation road profile with different grades of slope

For the determination of the driver's weights, the $Q$ value was first set to 1 and the vehicle simulation was evaluated with this single weight factor. Then weight $Q$ was decreased by a constant and the simulation was evaluated with the calculated $\Gamma$ values. The decreasing of the weight $Q$ was repeated until the square difference of the original velocity profile and the simulated velocity profile was minimal. The results imply that the driver uses a weight selection for $Q$ to be around 0.8 , thus in the behavior of the driver the minimization of the traveling time is of high importance, while the weighting of the road slope and velocity regulation are minimal compared to the automatic system. After the identification of the driver's weights, the look-ahead cruise control can be tuned to better fit the behavior of a human driver enhancing the comfort level of the system in this manner. The realization of the driver's behavior adaptation in the automatic system can be carried out by different methods. The simplest way is to adopt the driver's weighting function parameters $Q$ for the automatic system, and let the look-ahead optimization method calculate the optimal $\Gamma$ values with this fix parameter. In this case, the automatic system will degrade in terms of energy efficiency, but the traveling time will be closer to that of the human driver's. The consideration of the road slope will still be captured in the cruise control with a smaller weight, thus the energy consumption will be lower than that of a human driver's.

In order to determine the effect of weighting parameter $Q$ in the actuated force (braking and propulsion force) during the travel, the following analysis is evaluated. The simulated vehicle has been run on three different kinds of routes with the same profile, but with different slope angles. The flattest one contains slopes with a grade of less than 1 percent $(0.45 \mathrm{deg})$, the medium one with grades less than 3 percent $(1.35 \mathrm{deg})$, while the most hilly road contains slopes with grades of less than 5 percent $(2.25 \mathrm{deg})$, which is the maximum road slope permitted in motorway design. The profile of the $5 \mathrm{~km}$ road is shown in Fig. 4 .

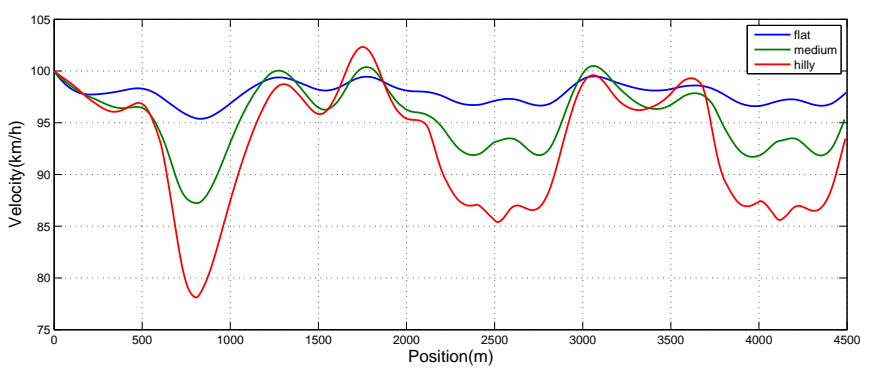

(a) Velocity profiles with $Q=0$

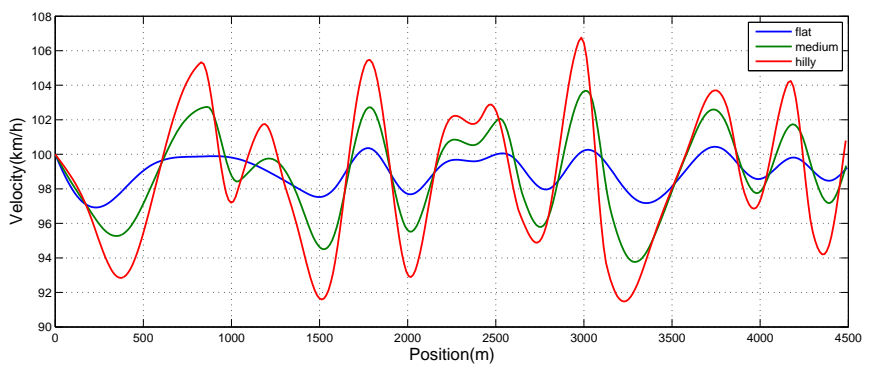

(b) Velocity profiles with $Q=1$

Fig. 5. Velocity profiles with different $Q$ values

The regulated maximum velocity on this road is $100 \mathrm{~km} / \mathrm{h}$. The effect of the weighting selection on the energy saving has been analyzed as follows. Weight $Q$ was set to different values $(0 ; 0.25 ; 0.5 ; 0.75 ; 1)$, and the automated vehicle was simulated on the three different roads while the optimization process calculated the optimal $\gamma_{i}$ values. From the simulated velocity and longitudinal actuated force data, the total energy consumption can be calculated as well as the traveling time. The simulated velocities are shown in Fig. 5 for the two terminal $Q$ values. As it is expected, the velocity difference from the speed limit on each road is increasing as the $Q$ weight tends to zero, and in parallel the difference increases as the grade of the slope is becoming higher.

In Fig. 6 the total energy consumption of the vehicle is shown for the three different roads and different $Q$ values set. As it can be observed, on a relatively flat road the total energy consumption of the automatic system is just slightly increasing with the increasing value of $Q$. On the medium slope and the hilly road, the difference in total energy consumption related to value $Q$ is more significant, however, even with $Q=0.75$ there is a sig- 


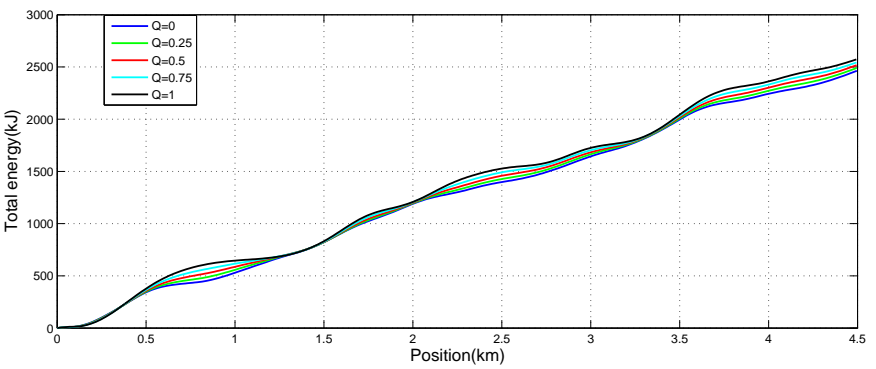

(a) Energy consumed on flat road

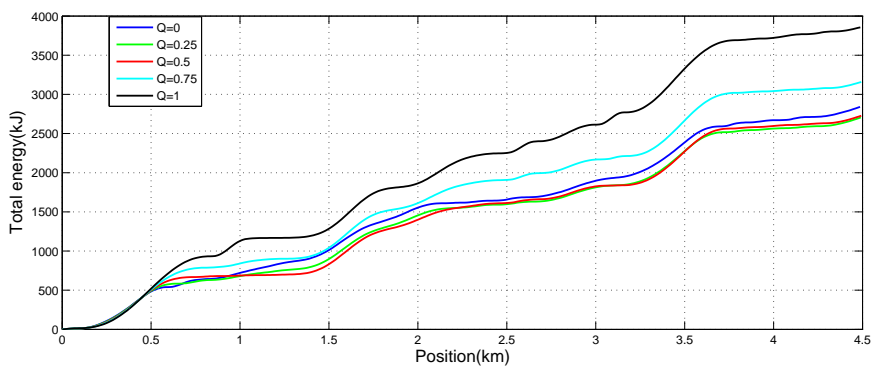

(b) Energy consumed on medium road

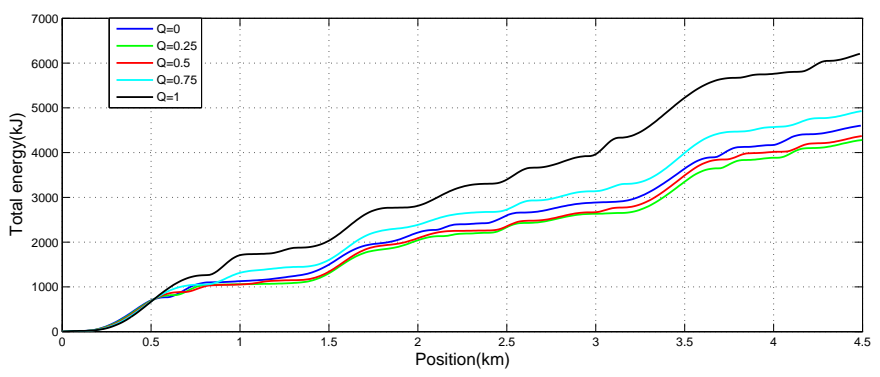

(c) Energy consumed on hilly road

Fig. 6. Total energy consumption with different $Q$ values

nificant amount of energy saved compared to the case when the road information is not considered $(Q=1)$. This result suggests that by setting the automatic system's weighting function $Q$ to an average value set by the driver,the traveling time may be closer to that of the human driver while the energy consumption can still be reduced significantly.

\subsection{Results with driver model}

With the above detailed driver model,the actual velocity of the vehicle is calculated during the operation of the look-ahead system. With the calculated velocity and acceleration data $Q_{i}$ weights are defined and added to the optimization process of the automatic system as an initial condition. In Fig.7(a) the velocity of the original automatic system and the velocity of modified system are compared, together with the velocity profile of the driver model. As it can be seen, the velocity profile of the original look-ahead system and that of the modified system differ greatly, the latter profile being closer to that suggested by the driver model. Note that by limiting the driver model suggested $Q$ weight, which is in the domain $Q \in[0,1]$, the abrupt behav-

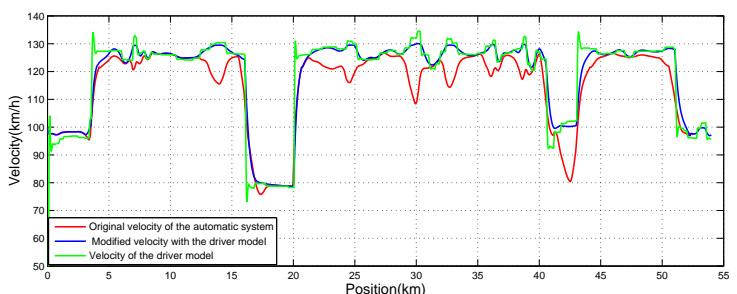

(a) Comparison of the original and the simulated velocity profiles

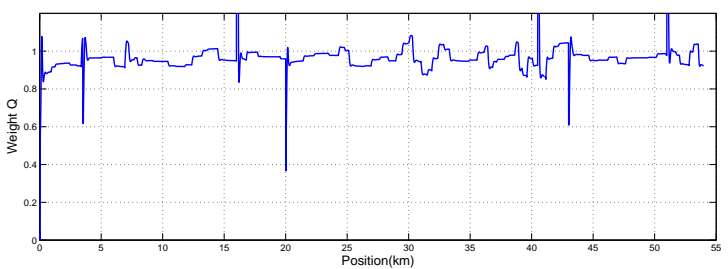

(b) Q weights set by the driver model

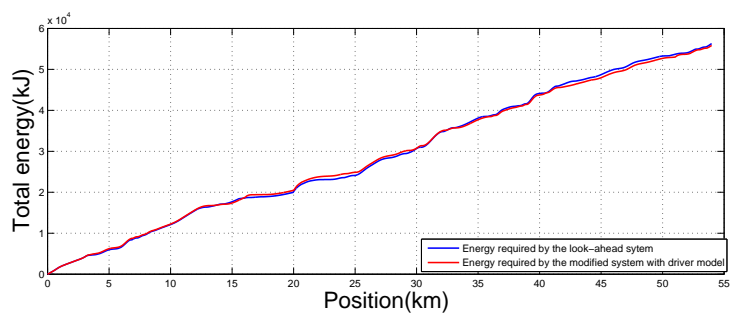

(c) Total energy consumption

Fig. 7. Velocity profile of original and modified system

ior of the driver has been smoothen by the automatic system. However, the tendency of the velocity may be more comfortable to the driver and the passengers. More interestingly, the total energy required for the journey has not changed notably by selecting the automatic system's velocity profile to be close to that of the driver, as it can be seen in Fig. 7 (b) .

\section{Summary}

The paper presented a control design method for velocity optimization with the consideration of road information, i.e terrain characteristics and velocity regulations. After a brief description of the driver's simulation environment, an optimization method was discussed, which regressively calculates the weighting factors possibly used by the driver instinctively. The optimization calculation was validated by simulating the automatic system's velocity profile with the use of the regressively calculated weights. From the results of the driver's simulation, the driver's weight selection was then mapped with this method and the automatic system's algorithm was tuned to fit better the driver's behavior. The effect of changing the weight parameter on the total energy consumption was also analyzed by simulation and calculation.

In the paper, a different process using a longitudinal velocity tracking driver model was also introduced for the tuning of the look-ahead system. The process of integrating a driver model in the velocity design method was also simulated and analyzed, resulting in a velocity design algorithm, which is more com- 
fortable for the passengers while preserving the energy saving benefits of the original look-ahead system.

\section{Acknowledgement}

The work is connected to the scientific program of the 'Development of quality-oriented and harmonized $\mathrm{R}+\mathrm{D}+\mathrm{I}$ strategy and functional model at BME' project. This project is supported by the the Hungarian Scientific Research Fund (OTKA) through grant No. CNK-78168 and by the New Hungary Development Plan (Project ID: TÁMOP-4.2.1/B-09/1/KMR-20100002) which are gratefully acknowledged. The research was supported by the grant TÁMOP- 4.2.2.B-10/1-2010-0009.

\section{References}

1 Trencséni B, Palkovics L, Driveline torque observer for heavy duty vehicle, Periodica Polytechnica Transportation Engineering 39 (2011), no. 2, 91-97, DOI 10.3311/pp.tr.2011-2.08.

2 Ivarsson M, Åslund J, Nielsen L, Look Ahead Control - Consequences of a Non-Linear Fuel Map on Truck Fuel Consumption, Proceedings of the Institution of Mechanical Engineers, Part D: Journal of Automobile Engineering 223 (2009), 1223-1238, DOI 10.1243/09544070JAUTO1131.

3 Nouveliere L, Braci M, Menhour L, Luu H T, Fuel consumption optimization for a city bus, UKACC Control Conference (2008).

4 Hellström E, Ivarsson M, Åslund J, Nielsen L, Look-aahead Control for Heavy Trucks to minimize Trip Time and Fuel Consumption, Control Engineering Practice 17 (2009), no. 2, 245-254, DOI 10.1016/j.conengprac.2008.07.005.

5 Sahlholm P, Johansson $\mathbf{K} \mathbf{H}$, Road grade estimation for look-ahead vehicle control using multiple measurement runs, Control Engineering Practice 18 (2010), 1328-1341, DOI 10.1016/j.conengprac.2009.09.007.

6 Németh B, Gáspár P, Road inclinations in the design of LPV-based adaptive cruise control, 18th IFAC World Congress (2011), 2202-2207.

7 Mihály A, Németh B, Gáspár P, Analysis of driver behavior related to look-ahead control, 13-th IFAC Symposium on Control in Transportation Systems 13 (2012), 268-273, DOI 10.3182/20120912-3-BG-2031.00056. Part 1.

8 Gill P E, Murray W, Wright M H, Practical Optimization, Academic Press, London UK, 1981.

9 Carlo Cacciabue P, Modelling Driver Behaviour in Automotive Environments, Springer, 2007.

10 Németh B, Gáspár P, Considering predicted road conditions in platoon control design, Periodica Polytechnica Transportation Engineering 39 (2011), no. 2, 69-75, DOI 10.3311/pp.tr.2011-2.04. 